\title{
Assessment of the right internal jugular vein cross-sectional area with different levels of positive end-expiratory pressure in patients with controlled ventilation during anesthesia
}

\author{
Young Woo Cho, Dae-Young Kim, Soo Jin Shin, and Kang-Il Kim \\ Department of Anesthesiology and Pain Medicine, Ulsan University Hospital, University of Ulsan College of Medicine, Ulsan, Korea
}

For drug administration, IV fluid administration, and if securing a peripheral vein is difficult, the internal jugular vein is used for the central venous catheter to secure an IV route and for central venous pressure monitoring. Theoretically, the bigger the cross-sectional area (CSA) of the internal jugular vein, the higher the success rate when cannulation is carried out, and the smaller the area, the higher the possibility of failure, increasing the risk of complications such as hemothorax, pneumothorax, and arterial puncture [1].

Most existing cannulation methods are attempted in the trendelenburg position with leg elevation, but positive end expiratory pressure (PEEP) is being introduced as an useful method for patients under control of this study is to find an effective PEEP level for the internal jugular vein cannulation for patients under controlled ventilation and who have difficulties with the trendelenburg position.

This study includes 20 American Society of Anesthesiology (ASA) Physical Status I or II patients between 24-83 year of age undergoing elective surgery that required central venous access. All patients received standardized induction of general anesthesia with penthotal sodium $5 \mathrm{mg} / \mathrm{kg}$ IV, lidocaine $60 \mathrm{mg}$ IV, rocuronium $0.8-1 \mathrm{mg} / \mathrm{kg}$ IV. The patients were tracheally intubated and their lungs mechanically ventilated using volume-control mode with tidal volume $6-8 \mathrm{ml} / \mathrm{kg}$, respiratory rate 10 breaths/min, inspiratory to expiratory ratio $1: 2$, PEEP 0 $\mathrm{cmH}_{2} \mathrm{O}$, and were maintained with $\mathrm{O}_{2}$-Air-Desflurane.

After anesthetic induction, the bed was placed in a level position and the patient was laid in the supine position, A 13 $\mathrm{MHz}$ linear transducer of Ultrasound (Esoate ${ }^{\circledR}$, Wing international, Italy) was placed vertically on the skin at the angular point where the medial sternocleidomastoid muscle and lateral sternocleidomastoid muscle meet, and the images of right internal jugular vein were acquired applying as little pressure as possible. (P0) The size of right internal jugular vein differs with each breath, and the largest size was recorded. Following the above method, PEEP 5 (P5), 10 (P10), 15 (P15) $\mathrm{cmH}_{2} \mathrm{O}$ and $10^{\circ}$ trendelenburg position $(\mathrm{T})$ were applied in sequence every two minutes. After the application, images of the right internal jugular vein were obtained and stored, and sizes compared (Fig. 1).

In the right internal jugular vein measurement, the size of the $10^{\circ}$ trendelenburg group $(\mathrm{T})$ and the PEEP groups (P5, P10, $\left.\mathrm{P} 15\right)$ increased statistically significantly compared to the comparison group (P0) (Table 1), Though the P5 group increased statistically significantly when compared to P0 group, it was statistically different from the $\mathrm{T}$ group. There was no significant difference of P10 and P15 compared with T.

Several methods are available to increase the size of CSA of the internal jugular vein, and the trendelenburg position or Valsalva method increases the CSA by promoting superior vena cava filling [2]. Other methods include PEEP, hepatic compression, humiming tone, skin traction, use of a small pillow under the head, increase of diameter by breathing. On the other hand, a compressed carotid artery or excessive extension of neck by high support under the shoulder will only decrease

Corresponding author: Dae-Young Kim, M.D., Department of Anesthesiology and Pain Medicine, Ulsan University Hospital, Junhwa-dong, Dong-gu, Ulsan 682-714, Korea. Tel: 82-52-250-7248, Fax: 82-52-250-7249, E-mail: kdyangel@naver.com

(c) This is an open-access article distributed under the terms of the Creative Commons Attribution Non-Commercial License (http:// creativecommons.org/licenses/by-nc/3.0/), which permits unrestricted non-commercial use, distribution, and reproduction in any medium, provided the original work is properly cited. 

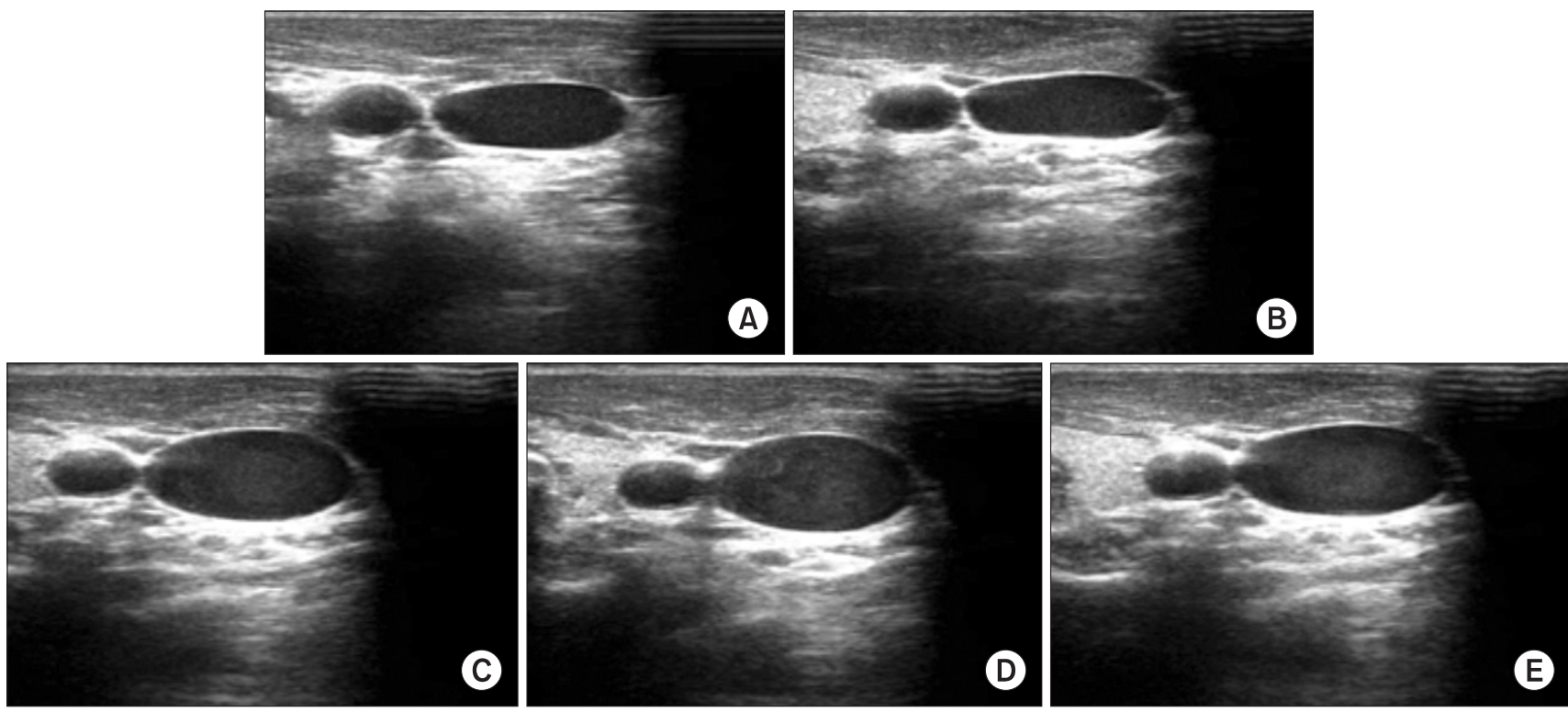

Fig. 1. Ultrasonic images of $\operatorname{PEEP}\left(0,5,10,15 \mathrm{cmH}_{2} \mathrm{O}\right)$ and $10^{\circ}$ trendelenburg position on the right internal jugular vein. (A) $\mathrm{PEEP} 0 \mathrm{cmH} \mathrm{H}_{2} \mathrm{O},(\mathrm{B})$ PEEP $5 \mathrm{cmH}_{2} \mathrm{O}$, (C) PEEP $10 \mathrm{cmH}_{2} \mathrm{O}$, (D) PEEP $15 \mathrm{cmH}_{2} \mathrm{O}$, (E) $10^{\circ}$ trendelenburg position.

Table 1. Right Internal Jugular Vein Measurements

\begin{tabular}{llll}
\hline & CSA $\left(\mathrm{cm}^{2}\right)$ & TD $(\mathrm{cm})$ & APD $(\mathrm{cm})$ \\
\hline P0 & $0.95 \pm 0.51^{\dagger}$ & $1.363 \pm 0.55^{\dagger}$ & $0.653 \pm 0.329^{\dagger}$ \\
P5 & $1.13 \pm 0.56^{*}$, & $1.454 \pm 0.596^{*}$ & $1.032 \pm 1.307^{*}$ \\
P10 & $1.39 \pm 0.62^{*}$ & $1.603 \pm 0.669^{*}$ & $0.872 \pm 0.392^{*}$ \\
P15 & $1.54 \pm 0.69^{*}$ & $1.635 \pm 0.661^{*}$ & $1.315 \pm 1.604^{*, \dagger}$ \\
T & $1.48 \pm 0.67^{*}$ & $1.653 \pm 0.692^{*}$ & $0.898 \pm 0.389^{*}$ \\
\hline
\end{tabular}

CSA: cross-sectional area $\left(\mathrm{cm}^{2}\right)$, TD: transverse diameter $(\mathrm{cm})$, APD: anterposterior diameter $(\mathrm{cm})$, PEEP: positive end expiratory pressure $\left(\mathrm{cmH}_{2} \mathrm{O}\right)$, P0: PEEP $0 \mathrm{cmH}_{2} \mathrm{O}$, P5: PEEP $5 \mathrm{cmH}_{2} \mathrm{O}$, P10: PEEP 10 $\mathrm{cmH}_{2} \mathrm{O}, \mathrm{P} 15$ : PEEP $15 \mathrm{cmH}_{2} \mathrm{O}, \mathrm{T}: 10^{\circ}$ trendelenburg position. Values are mean $\pm \mathrm{SD} .{ }^{*} \mathrm{P}<0.05$ compared with $\mathrm{P} 0,{ }^{\dagger} \mathrm{P}<0.05$ compared with $\mathrm{T}$.

CSA [3]. Hepatic compression, humming tone and Valsalva are not practical in anesthetized patients and therefore further evaluation is needed [4]. The trendelenburg position increased the height between the right atrium and the right internal jugular vein and decreased the return to the superior vena cava. Up to $10^{\circ}$, CSA was increased, but increasing to $20^{\circ}$ did not make any difference. Rather than using just only the trendelenburg position, when used with Valsava or hepatic compression, the CSA was increased more [5]. However, if the patient is obese, abdominal contents are raised, so it is difficult to ventilate or oxygenate [5].

PEEP can be applied easily to patients under controlled ventilation, and also in cases where the trendelenburg position cannot be used i.e. spinal surgery that requires the patient to be under anesthesia on a transfer bed and moved over to the operating table, or where surgery needs to be performed in the prone position, or when the central venous catheter cannulation is carried out in the intensive care unit. However, vital signs may deteriorate if PEEP is used for the patients with increased intracranial pressure and poor heart function, and with seriously dehydrated patients. In addition, it could expand the upper lung field, increasing the risk of pneumothorax [4]. For ASA I \& II patients who participated in this experiment, PEEP increased the size of the CSA as much as the trendelenburg position, and although there were some changes in the vital signs, they were not serious.

In conclusion, the results of this study show that, if cannulation of the central venous catheter is carried out in a patient under controlled ventilation, above $10 \mathrm{cmH}_{2} \mathrm{O}$ PEEP method is easy and thought to increase the success rate as it increases the CSA as much as the trendelenburg position. We expect that the success rate is higher when combining trendelenburg position and PEEP. In cases where the patient is seriously dehydrated or has poor heart function, vital signs will need to be carefully monitored.

\section{References}

1. Kusminsky RE. Complication of central venous catherization. J Am Coll Surg 2007; 204: 681-96.

2. Hollenbeck KJ, Vander Schuur BM, Tulis MR, Mecklenburg BW, Gaconnet CP, Wallace SC, et al. Brief report: effects of positive endexpiratory pressure on internal jugular vein cross-sectional area in anesthetized adults. Anesth Analg 2010; 110: 1669-73.

3. Chung IS, Kwon MA, Hwang HY, Park JH, Yeo JS, Kim CS, et al. The examination of internal jugular vein and carotid artery in trendelenburg position with head rotation; a prospective, rando- 
mized study. Korean J Anesthesiol 2006; 51: 11-6.

4. Marcus HE, Bonkat E, Dagtekin O, Schier R, Petzke F, Wippermann J, et al. The impact of Trendelenburg position and positive endexpiratory pressure on the internal jugular cross-sectional area. Anesth Analg 2010; 111: 432-6.
5. Lobato EB, Florete OG Jr, Paige GB, Morey TE. Cross-sectional area and intravascular pressure of the right internal jugular vein during anesthesia: effects of Tredelenburg position, positive intrathoracic presure, and hepatic compression. J Clin Anesth 1998; 10: 1-5. 